\title{
Understanding adherence in virally suppressed and unsuppressed human immunodeficiency virus-positive urban patients on second-line antiretroviral treatment
}

\begin{tabular}{|c|c|}
\hline \multicolumn{2}{|c|}{$\begin{array}{l}\text { Authors: } \\
\text { Siphamandla B. Gumede } \\
\text { Willem D.F. Venter } \\
\text { Samanta T. Lalla-Edward }\end{array}$} \\
\hline $\begin{array}{l}\text { Affiliations: } \\
{ }^{1} \text { Ezintsha, a su } \\
\text { Wits Reproduc } \\
\text { and HIV Institu } \\
\text { of the Witwat } \\
\text { Johannesburg, }\end{array}$ & $\begin{array}{l}\text { b-division of } \\
\text { ctive Health } \\
\text { ute, University } \\
\text { ersrand, } \\
\text { South Africa }\end{array}$ \\
\hline $\begin{array}{l}{ }^{2} \text { Department } \\
\text { Interdisciplina } \\
\text { Science, Publi } \\
\text { Utrecht Unive } \\
\text { The Netherlan }\end{array}$ & $\begin{array}{l}\text { ff } \\
\text { ry Social } \\
\text { c Health, } \\
\text { rsity, Utrecht, } \\
\text { ids }\end{array}$ \\
\hline $\begin{array}{l}\text { Corresponding } \\
\text { Siphamandla C } \\
\text { sgumede@car }\end{array}$ & $\begin{array}{l}\text { g author: } \\
\text { Sumede, } \\
\text { tafrica.org }\end{array}$ \\
\hline $\begin{array}{l}\text { Dates: } \\
\text { Received: } 24 \mathrm{~N} \\
\text { Accepted: } 13 \mathrm{~J} \\
\text { Published: } 11\end{array}$ & $\begin{array}{l}\text { May } 2020 \\
\text { lune } 2020 \\
\text { Aug. } 2020\end{array}$ \\
\hline $\begin{array}{l}\text { How to cite th } \\
\text { Gumede SB, V } \\
\text { Lalla-Edward S } \\
\text { Understanding } \\
\text { virally suppres } \\
\text { unsuppressed } \\
\text { immunodefici } \\
\text { positive urban } \\
\text { second-line an } \\
\text { treatment. S A } \\
\text { 2020;21(1), a1 } \\
\text { doi.org/10.41 } \\
\text { v21i1.1107 }\end{array}$ & $\begin{array}{l}\text { is article: } \\
\text { enter WDF, } \\
\text { iT. } \\
\text { s adherence in } \\
\text { sed and } \\
\text { human } \\
\text { ency virus- } \\
\text { patients on } \\
\text { tiretroviral } \\
\text { fr J HIV Med. } \\
\text { 107. https:// } \\
\text { 02/sajhivmed. }\end{array}$ \\
\hline $\begin{array}{l}\text { Copyright: } \\
\text { (C) 2020. The A } \\
\text { Licensee: AOS } \\
\text { is licensed und } \\
\text { Creative Comn } \\
\text { Attribution Lic }\end{array}$ & $\begin{array}{l}\text { luthors. } \\
\text { IS. This work } \\
\text { der the } \\
\text { nons } \\
\text { ense. }\end{array}$ \\
\hline 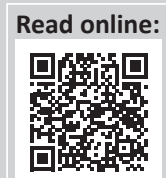 & $\begin{array}{l}\text { Scan this QR } \\
\text { code with your } \\
\text { smart phone or } \\
\text { mobile device } \\
\text { to read online. }\end{array}$ \\
\hline
\end{tabular}

Background: Understanding antiretroviral therapy (ART) adherence may assist in designing effective support interventions.

Objectives: This study elicited perspectives on how to promote treatment adherence from virologically suppressed and unsuppressed patients receiving second-line ART.

Methods: This was a cross-sectional study conducted with randomly selected patients active on second-line ART, from five public health facilities in the Johannesburg inner city. Data were collected on demographics, clinical information, participant's experiences and ART knowledge. Virological failure was defined as exceeding 1000 copies $/ \mathrm{mL}$.

Results: The study sample comprised 149 participants; of which $47.7 \%(n=71)$ were virally unsuppressed and $69.1 \%(n=103)$ were women; the median age of the participants was 42 years (interquartile range [IQR] 36-47 years). Experiencing medication-related difficulties in taking second-line ART ( $p=0.003)$, finding second-line regimen more difficult to take than a first-line regimen $(p=0.001)$ and experiencing side effects $(p<0.001)$ were all subjective predictors of virological failure. Participants' recommendations for improving adherence included the introduction of a single tablet regimen $(31.6 \%, n=55)$, reducing the dosage to once daily $(26.4 \%, n=46)$ and reducing the pill size for second-line regimen $(4.0 \%, n=7)$.

Conclusion: The results of this study highlight the importance of improving patients' knowledge about adherence and motivation to continue ART use despite the persistence of side effects and difficulties with taking medication.

Keywords: adherence; viral load suppression; virological failure; antiretroviral therapy; South Africa.

\section{Introduction}

The World Health Organization (WHO) defines adherence as the degree to which a patient is able to follow a treatment plan and take medication at prescribed times. ${ }^{1}$

Factors that affect treatment adherence include changes in daily routines, forgetting to take medication, side effects, depression, being away from home, comorbidity, lack of knowledge and desire to take treatment. ${ }^{2,3,4}$ In addition, patients experiencing financial constraints, social issues such as the fear of disclosure and lack of understanding of treatment benefits are more prone to non-adherence to treatment and illnesses. ${ }^{5,6}$ Some studies have reported disclosure and relationship with the person being disclosed to as predictors of adherence. ${ }^{7,8}$

The South African government's adherence promotion strategies include routine viral load monitoring, adherence counselling, pill counting, adherence clubs and routine completion of clinical stationery. ${ }^{9,10,11}$ Despite all these antiretroviral therapy (ART) adherence strategies being implemented, treatment failure amongst patients on first- and second-line ART remains an issue. ${ }^{12}$ Lapses in ART medication adherence can lead to viral rebound with ongoing immunosuppression and viral resistance. ${ }^{1,13,14}$ However, not much is understood about the perspectives of patients regarding adherence challenges. ${ }^{15,16}$

Therefore, this study sought to describe and obtain perspectives on treatment adherence from two separate groups of patients on second-line therapy, those who were suppressed and those who were not, to understand treatment-taking behaviour. 


\section{Materials and methods Study design and setting}

This was a cross-sectional study conducted between July and August 2018 in a sub-population of patients receiving secondline ART at the end of June 2018. Five public health facilities in inner-city Johannesburg (two hospitals, one community health centre and two primary healthcare clinics) were included in the study.

\section{Study population}

The study population comprised patients aged 18 years and older who were on second-line ART for at least 1 month or longer.

\section{Data collection}

\section{Sample selection and recruitment}

We randomly sampled $10 \%$ of the population of 1500 eligible patients. The total number of active patients on second-line treatment per facility was divided by the total sample size $(n=150)$ to determine the interval that needed to be used to select the eligible patients. Using this formula, every $n$th (different for each facility) record from the register or list of active patients on second-line treatment in each facility was selected and recruited to the study until the facility sample size was reached. Once the eligible patients were identified, they were invited to participate in the study in one of the two ways: telephonically or in facility recruitment where researchers met them at the facility during their scheduled clinic visit. For the patients who refused to participate in the study, the next $n$th patient was recruited.

\section{Data collection, tool and variables}

A pretested semi-structured questionnaire was used, which consisted of five sections: (1) demographic data, (2) comorbidity information, (3) human immunodeficiency virus (HIV) diagnosis and care information, (4) experiences on the first-line regimen and adherence and (5) experiences on second-line regimen and adherence. Information collected included demographic information (facility name, sex, age, relationship status, employment status and education level), comorbidity information, experiences on both first- and second-line treatment, disclosure information, duration on ART, reasons for starting ART, side effects, self-reported treatment interruptions, challenges with taking second-line treatment, treatment supporter information and insights into how adherence could be improved.

\section{Questionnaire administration}

Data were collected by the principal investigator and a trained research assistant. The interviews were conducted in English as it was the most commonly spoken language in the study setting and all participants could speak it.

\section{Data entry, cleaning and analysis}

Data were captured into REDCap immediately after interviews were conducted. The research team conducted data clean-up by running data quality checks in REDCap and STATA (quantitative data). For the closed-ended questions, we assessed the association between outcome variables and selected sociodemographic and health-related characteristics. Pearson's chisquared test was used to assess trend associations between categorical variables. Continuous data were summarised and analysed using the median and interquartile ranges (IQRs). Logistic and multiple logistic regression models (bivariate and multivariate logistic regression) were built for key outcome variables, such as viral load, difficulties in taking second-line regimen and side effects, to identify independent predictors. We reported unadjusted and adjusted odds ratios (ORs), 95\% confidence interval (CI) and $p$-values - $p$-values that were less than 0.05 were considered significant. Open-ended questions were analysed using qualitative data analysis methods. Data were coded and thematic analysis was performed. Where appropriate, quotations have been included to support the reported results.

\section{Ethical consideration}

Ethical approval to conduct the study was obtained from the University of the Witwatersrand Human Research Ethics Committee (ethical clearance number: M170691). Approval was also granted by the Johannesburg Health District (DRC Ref No. 2017-08-003 and NHRD Ref No. GP_201708_030). Participants were informed that participation in the study was voluntary and that refusal would not affect their relationship with their healthcare provider or facility. All patients who agreed to participate in the study signed an informed consent form. To ensure confidentiality, there were no linkages between the data collected in the questionnaire and the patients' clinic information. Participants were reimbursed for their travel.

\section{Results}

\section{Sample characteristics}

A total of 150 out of 1500 active patients on second-line ART across the five public health facilities were interviewed (69.1\%, $n=103$ women). During the quality checking processes, we found that one of the participants was younger than 18 years and was subsequently omitted from the analysis. The median age of the participants was 42 years (IQR 36-47 years). Most of the participants were single $(38.1 \%, n=57)$; $30.2 \%(n=45)$ participants were married. Nearly two-thirds of the participants were born in South Africa $(61.1 \%, n=91$ ), whilst almost one-third of the participants were born in Zimbabwe $(32.9 \%, n=49)$. The majority $(87.2 \%, n=130)$ of participants had completed at least their secondary or high school-level education. A minority $(8.1 \%, n=12)$ of participants had completed tertiary qualifications; $4.7 \%(n=7)$ participants had never attended a school. Of the total participants, $45.6 \%$ $(n=68)$ were unemployed. The majority of participants were identified as Christian $(87.9 \%, n=131)$. Hypertension $(65.1 \%$, $n=28 / 43)$, diabetes $(9.3 \%, n=4 / 43)$ and hypercholesterolaemia (9.3\%, $n=4 / 43)$ were the most common concomitant conditions reported by the participants. The average distance travelled to reach a health facility was $5 \mathrm{~km}$ (IQR: $2 \mathrm{~km}-15$ $\mathrm{km})$, with $57.7 \%(n=86)$ participants travelling $5 \mathrm{~km}$ or less to reach the health facility. 
TABLE 1: Socio-demographic and treatment-taking characteristics disaggregated by virological status of second-line participants in five health facilities in the Johannesburg inner city.

Variable

Total patient or participants recruited

\begin{tabular}{|c|c|c|}
\hline \multirow[t]{2}{*}{ Total $N=149(100)$} & \multicolumn{2}{|c|}{ Virological status } \\
\hline & $\begin{array}{c}\text { Suppressed (VLS), } \\
78(52.3)\end{array}$ & $\begin{array}{c}\text { Unsuppressed (VLF), } \\
71(47.7)\end{array}$ \\
\hline$\%$ & $\%$ & $\%$ \\
\hline
\end{tabular}

\section{Facility}

Charlotte Maxeke Johannesburg Academic Hospital

$32 \quad 21.5$

Hillbrow Community Health Centre

South Rand Hospital

Primary Healthcare Clinics (Malvern Clinic Yeoville Clinic $\dagger$ )

$86 \quad 57.7$

$21 \quad 14.1$

Sex

Female

10

57.7
14.1

6.7

19
43
16
0

24.4

55.1

20.5

0.0

Male

$103 \quad 69.1$

69.1

51

46

30.9

51
27

\section{4}

34.6

$\begin{array}{cc}13 & 18.3 \\ 43 & 60.6 \\ 5 & 7.0 \\ 10 & 14.1\end{array}$

Age, Median (42, IQR: 35-47) (years)

$<30$

30-39

40-45

$45+$

$11 \quad 7.4$

$7.4 \quad 3$

\section{3}

34.9

28.9

43

Country of birth

South Africa

Zimbabwe

Other

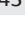

28.9

24

24
25

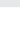

61.1

26

61.1
32.9

6.0

$\begin{array}{lll}3.9 & 8 & 11.3 \\ 30.8 & 28 & 39.4 \\ 32.1 & 18 & 25.4 \\ 33.3 & 17 & 23.9\end{array}$

Home language

Zulu

Ndebele

Xhosa

Sotho

Other

49

42

53.9

41.0

5.1

49

69.0

0.085

\section{Relationship status}

Married

Cohabiting

Single

Other

9

32

62

36

41.6

24.2

8.1

8.1

12

27

18.1

4

$17 \quad 23.9$

$17 \quad 23.9$

$\begin{array}{lll}41.0 & 30 & 42.3\end{array}$

$\begin{array}{lll}26.9 & 15 & 21.1\end{array}$

$\begin{array}{lll}7.7 & 6 & 8.5\end{array}$

$\begin{array}{lll}6.4 & 7 & 9.9\end{array}$

18.0

$45 \quad 30.2$

$38 \quad 25.5$

$57 \quad 38.3$

9

38.3
6.0

Highest education level completed

6.0

Never went to school

Secondary or high school

Tertiary

Employment status

Employed

Unemployed

Othert

$130 \quad 87.2$

12

8.1

8.1

$65 \quad 43.6$

43.6
45.6

10.7

16

Religion

Christian

Ancestral/traditional

Other

$131 \quad 87.9$

87.9
5.4

10

5.4
6.7

Comorbidity

Yes

No

106

28.9

71.1

32
21
6
5
14

14

13

9.9
18.3

Distance travelled to the facility,

Median (5, IQR: 2-15)

$5 \mathrm{~km}$ or less

$6 \mathrm{~km}-10 \mathrm{~km}$

$11 \mathrm{~km}-20 \mathrm{~km}$

Above $20 \mathrm{~km}$

Who was the first person to whom you disclosed your HIV status?

Partner

Family or relative member

Friend

How long did it take you to disclose your HIV status?

1 week

1-2 weeks

3-4 weeks

More than 4 weeks

Never disclosed

$\begin{array}{ll}86 & 57.7 \\ 21 & 14.1 \\ 23 & 15.4 \\ 19 & 12.8\end{array}$

28
16
30
4

35.9

20.5

38.5

5.1

$22 \quad 31.0$

$27 \quad 38.0$

5

7.0

$\begin{array}{ccc}5.1 & 3 & 4.2 \\ 88.5 & 61 & 85.9 \\ 6.4 & 7 & 9.9\end{array}$

9.9

$\begin{array}{lll}47.4 & 28 & 39.4\end{array}$

$\begin{array}{lll}47.3 & 35 & 49.3\end{array}$

$\begin{array}{lll}10.3 & 8 & 11.3\end{array}$

$85.9 \quad 64 \quad 90.1$

$\begin{array}{ccc}85.9 & 64 & 90.1 \\ 9.0 & 1 & 1.4\end{array}$

5.1

4

5.1

1
6

29.5

70.5

20
51

8.5

51

28.2

71.8

1.3
90.1
1.4
8.5

28.2
71.8

$\begin{array}{ccc}57.7 & 41 & 57.8 \\ 15.4 & 9 & 12.7\end{array}$

$\begin{array}{ccc}15.4 & 9 & 12.7 \\ 12.8 & 13 & 18.3\end{array}$

$\begin{array}{ccc}14.1 & 8 & 18.3 \\ & 11.3\end{array}$

$\begin{array}{llllll}70 & 47.0 & 33 & 42.3 & 37 & 52.1\end{array}$

$\begin{array}{llllll}77 & 33 & 42.3 & 37 & 52.1 \\ & 51.7 & 43 & 55.1 & 34 & 47.9\end{array}$

$\begin{array}{cccccc}77 & 51.7 & 43 & 55.1 & 34 & 47.9 \\ 2 & 1.3 & 2 & 2.6 & 0 & 0.0\end{array}$

$\begin{array}{cccc}94 & 63.1 & 48 & 61.5 \\ 8 & 5.4 & 4 & 5 \\ 4 & 2.7 & 4 & 5 \\ 42 & 28.2 & 22 & 28.2 \\ 1 & 0.7 & 0 & 0.0\end{array}$

\begin{tabular}{cc}
61.5 & 46 \\
5.1 & 4 \\
5.1 & 0 \\
28.2 & 20 \\
0.0 & \\
\hline
\end{tabular}

\begin{tabular}{ccc} 
& & 0.306 \\
46 & 64.8 \\
4 & 5.6 \\
0 & 0.0 \\
20 & 28.2 \\
1 & 1.4 \\
\hline
\end{tabular}


TABLE 1(continuous): Socio-demographic and treatment-taking characteristics disaggregated by virological status of second-line participants in five health facilities in the Johannesburg inner city.

\begin{tabular}{|c|c|c|c|c|c|c|c|}
\hline \multirow{3}{*}{$\begin{array}{l}\text { Variable } \\
\text { Total patient or participants recruited }\end{array}$} & \multirow{2}{*}{\multicolumn{2}{|c|}{ Total $N=149(100)$}} & \multicolumn{4}{|c|}{ Virological status } & \multirow[t]{3}{*}{$p$} \\
\hline & & & \multicolumn{2}{|c|}{$\begin{array}{c}\text { Suppressed (VLS), } \\
78(52.3)\end{array}$} & \multicolumn{2}{|c|}{$\begin{array}{l}\text { Unsuppressed (VLF), } \\
71(47.7)\end{array}$} & \\
\hline & $n$ & $\%$ & $n$ & $\%$ & $n$ & $\%$ & \\
\hline Do you have anyone supporting you in taking your ARVs currently? & & & & & & & 0.755 \\
\hline Yes & 118 & 79.2 & 61 & 78.2 & 57 & 80.3 & \\
\hline No & 31 & 20.8 & 17 & 21.8 & 14 & 19.7 & \\
\hline $\begin{array}{l}\text { Do you feel the current regimen is difficult to take compared to the previous } \\
\text { regimen(s)? }\end{array}$ & & & & & & & $0.001 *$ \\
\hline Yes & 78 & 52.3 & 31 & 39.7 & 47 & 66.2 & \\
\hline No & 71 & 47.7 & 47 & 60.3 & 24 & 33.8 & \\
\hline Have you been experiencing difficulties in taking the current regimen? & & & & & & & $0.003 *$ \\
\hline Yes & 57 & 38.3 & 21 & 26.9 & 36 & 50.7 & \\
\hline No & 92 & 61.7 & 57 & 73.1 & 35 & 49.3 & \\
\hline Have you experienced any side effects since you switched regimens or drugs? & & & & & & & $<0.001 *$ \\
\hline Yes & 71 & 47.7 & 26 & 33.3 & 45 & 63.4 & \\
\hline Have you ever stopped taking the current regimen for over a month? & & & & & & & 0.728 \\
\hline Yes & 5 & 3.4 & 3 & 3.8 & 2 & 2.8 & \\
\hline No & 144 & 96.6 & 75 & 96.2 & 69 & 97.2 & \\
\hline Have you ever felt like stopping the current regimen or drugs completely? & & & & & & & 0.642 \\
\hline Yes & 15 & 10.1 & 7 & 9.0 & 8 & 11.3 & \\
\hline No & 134 & 89.9 & 71 & 91.0 & 63 & 88.7 & \\
\hline Have you ever felt like switching current regimen or drugs for something else? & & & & & & & 0.142 \\
\hline Yes & 58 & 38.9 & 26 & 33.3 & 32 & 45.1 & \\
\hline No & 91 & 61.1 & 52 & 66.7 & 39 & 54.9 & \\
\hline
\end{tabular}

$N$, number; VLS, virological suppression; $n$, number; VLF, virological failure; $p$, probability value; IQR, interquartile range; km, kilometre; ARVs, antiretrovirals; HIV, human immunodeficiency virus.

$\dagger$, Second-line patients are often managed at a higher level of facility.

$\$$, People not looking for employment at that time, for example, students and housewives.

$*, p<0.05$.

\section{Socio-demographic and virological status}

Table 1 shows the socio-demographic characteristics disaggregated by virological status. Nearly half $(47.7 \%$, $n=71$ ) of the participants interviewed had virological failure (VLF), with women accounting for $73.2 \%(n=52)$. With regard to age, of the total unsuppressed participants, $39.4 \%(n=28)$ were between 30 and 39 years. Of the participants with virological suppression (VLS), 29.5\% $(n=$ $23)$ had comorbidity compared to VLF participants $(28.2 \%$, $n=20)$.

\section{Disclosure, treatment support and virological status}

Almost all the participants' first disclosure of their HIV status was to a partner or relative $(98.7 \%, n=147$ combined). More VLS participants $(55.1 \%, n=43)$ disclosed about their HIV status to a family member first, whilst most VLF participants $(52.1 \%, n=37)$ chose to disclose to their partners first. Almost no disclosure to friends was reported. Disclosure did not show any statistical significance. Participants typically $(63.1 \%, n=94)$ disclosed within 1 week after HIV diagnoses, with more VLF participants reporting early disclosure than VLS participants $(64.8 \%, n=46$ vs. $61.5 \%, n=48)$. Most participants $(79.2 \%, n=118)$ had treatment supporters (VLF $=$ $80.3 \%, n=57$; VLS $=78.2 \%, n=61)$. Whilst $10.1 \%(n=15)$ of participants felt like stopping treatment completely at some point, only $3.4 \%(n=5)$ stopped treatment for longer than 1 month, more in VLS participants, although this was not statistically significant.

\section{Factors of virological failure and adherence}

Overall, there were more participants $(52.3 \%, n=78)$ who felt that taking a second-line regimen was difficult compared to the first-line regimen, with the VLF group $(66.2 \%, n=47$, $p=0.001$ ) predominantly reporting this challenge. Generally, $38.3 \%(n=57 / 149)$ experienced difficulties in taking the second-line regimen $(p=0.003)$. Of these, about two-thirds $(63.2 \%, n=36)$ were VLF participants $(p=0.003)$. Just under half $(47.7 \%, n=71 / 149)$ of the participants experienced side effects whilst taking their second-line regimen, and of these, $63.4 \%(n=45)$ were VLF participants $(p<0.001)$.

Table 2 presents results from both bivariate and multivariate logistic regression analysis. No association was detected between VLF and relationship status in bivariate analysis. However, in multivariate analysis, participants who cohabit were three times more likely to have a VLF than those who are married (adjusted odds ratios [AORs] 3.1, 95\% CI $=1.1-$ $8.9 ; p=0.035$ ). Unemployed participants were two and a half times more likely to have treatment-related side effects compared to employed participants (AOR 2.5, 95\% CI $=1.1$ 5.7; $p=0.023$ ). Results for age did not show any statistical significance but older people were less likely to be unsuppressed.

\section{Treatment-taking behaviour}

Table 3 presents the reported treatment behaviour of the participants for the duration of receiving ART. There were 


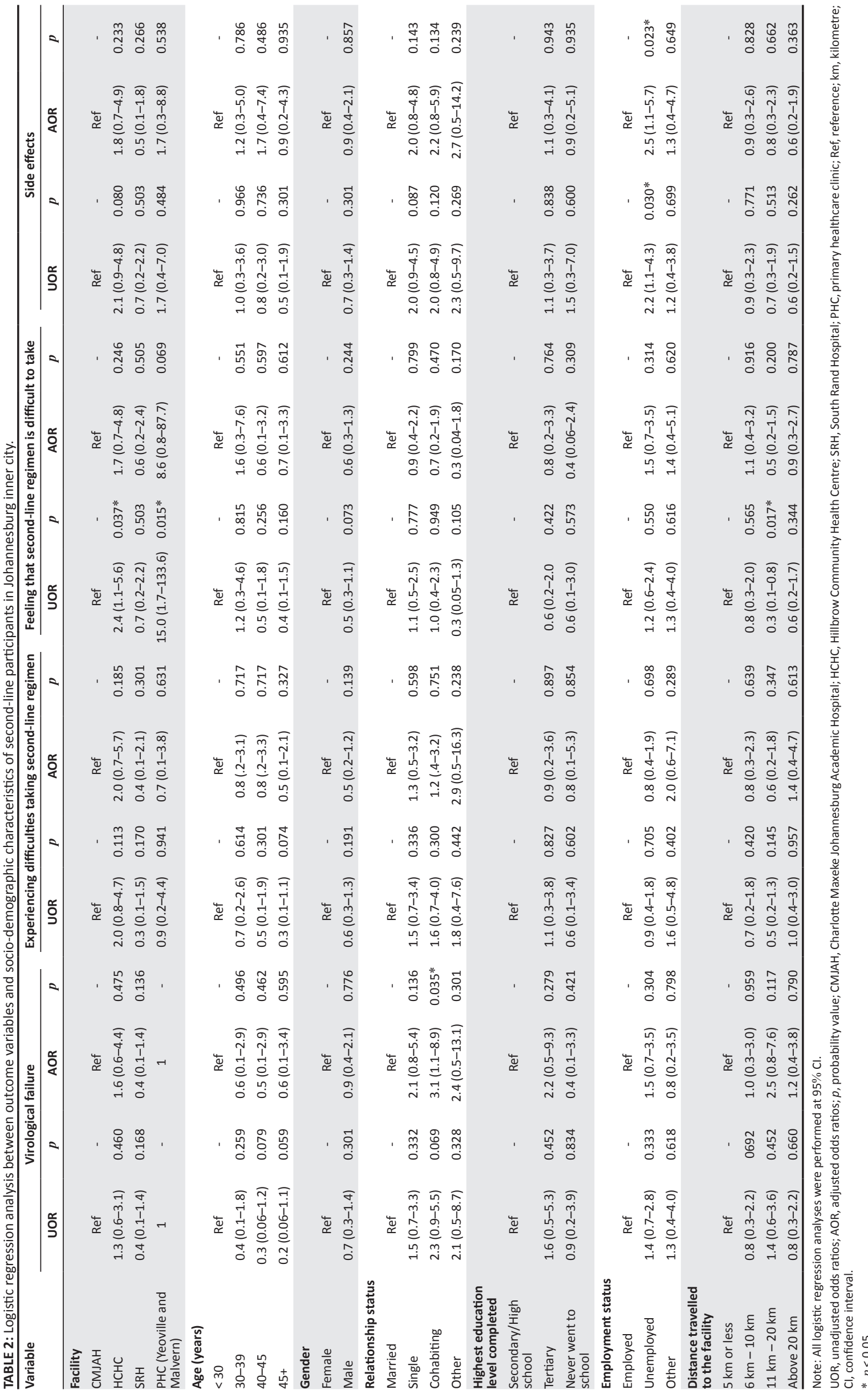


TABLE 3: History of antiretroviral treatment-taking behaviour.

\begin{tabular}{|c|c|c|c|c|c|c|c|c|c|}
\hline \multirow[t]{4}{*}{ Variable } & \multicolumn{3}{|c|}{ First-line treatment } & \multicolumn{6}{|c|}{ Second-line treatment } \\
\hline & \multirow[t]{2}{*}{$\begin{array}{c}\text { Treatment } \\
\text { interruption }\end{array}$} & \multirow[t]{2}{*}{$\begin{array}{l}\text { No treatment } \\
\text { interruption }\end{array}$} & \multirow[t]{2}{*}{$\begin{array}{l}\text { Did not report on } \\
\text { treatment interruption }\end{array}$} & \multicolumn{2}{|c|}{ Treatment interruption } & \multicolumn{2}{|c|}{ No treatment interruption } & \multicolumn{2}{|c|}{$\begin{array}{l}\text { Did not report on } \\
\text { treatment interruption }\end{array}$} \\
\hline & & & & VLS & VLF & VLS & VLF & VLS & VLF \\
\hline & 52 & 91 & 6 & 5 & 8 & 67 & 56 & 6 & 7 \\
\hline \multicolumn{10}{|l|}{ Medication alert } \\
\hline Alarm & 21 & 53 & 2 & 1 & 2 & 21 & 13 & 2 & 1 \\
\hline TV & 5 & 9 & - & 1 & 1 & 3 & 1 & - & - \\
\hline Memory & 14 & 14 & 2 & 1 & 4 & 30 & 30 & 3 & 4 \\
\hline Family & 7 & 9 & 1 & 1 & - & 2 & 4 & - & - \\
\hline Did not report & 5 & 6 & 1 & 1 & 1 & 11 & 8 & 1 & 2 \\
\hline \multicolumn{10}{|l|}{ Daily frequency } \\
\hline Once & 16 & 37 & 1 & - & 2 & 5 & 1 & 1 & 1 \\
\hline Twice & 33 & 52 & 4 & 5 & 6 & 60 & 55 & 5 & 5 \\
\hline Did not report & 3 & 2 & 1 & - & - & 2 & - & - & 1 \\
\hline Cupboard & 31 & 57 & 3 & 4 & 7 & 39 & 35 & 3 & 5 \\
\hline Handbag & 14 & 22 & 1 & - & - & 18 & 17 & 1 & 1 \\
\hline Cooler bag or fridge & 1 & 1 & 1 & - & - & - & 1 & 1 & - \\
\hline Pill box & 3 & 7 & - & - & - & 8 & 3 & - & - \\
\hline Other & 1 & 1 & - & - & 1 & - & - & 1 & - \\
\hline Did not report & 2 & 3 & 1 & 1 & - & 2 & - & - & 1 \\
\hline
\end{tabular}

ART, antiretroviral treatment; VL, viral load; VLS, viral load suppression; VLF, viral load failure; TV, television.

TABLE 4: Logistic regression analysis between virological failure, regimens (first-line and second-line regimen) and treatment-taking behaviour indicators in health facilities in Johannesburg inner city.

\begin{tabular}{|c|c|c|c|c|c|c|c|c|c|c|c|c|}
\hline \multirow[t]{2}{*}{ Variable } & \multicolumn{4}{|c|}{ Virological failure } & \multicolumn{4}{|c|}{ First-line interruption } & \multicolumn{4}{|c|}{ Second-line interruption } \\
\hline & UOR & $p$ & AOR & $p$ & UOR & $p$ & AOR & $p$ & UOR & $p$ & AOR & $p$ \\
\hline \multicolumn{13}{|l|}{ Medication alert } \\
\hline Alarm & 1 & - & 1 & - & 1 & - & 1 & - & 1 & - & 1 & - \\
\hline TV & $0.8(0.1-4.6)$ & 0.756 & $0.8(0.1-5.5)$ & 0.837 & $1.3(0.4-4.4)$ & 0.634 & $1.7(0.5-5.7)$ & 0.429 & $2.8(0.4-19.1)$ & 0.284 & $2.1(0.2-18.2)$ & 0.497 \\
\hline Memory & $1.7(0.8-3.7)$ & 0.196 & $1.7(0.8-3.8)$ & 0.194 & $2.8(1.2-6.6)$ & $0.023 *$ & $2.6(1.0-6.4)$ & $0.042 *$ & $1.1(0.4-3.3)$ & 0.818 & $1.1(0.3-3.7)$ & 0.859 \\
\hline Family & $1.9(0.4-8.1)$ & 0.398 & $2.1(0.5-9.8)$ & 0.339 & $2.7(0.9-7.9)$ & 0.069 & $2.7(0.9-8.4)$ & 0.080 & $0.7(0.07-6.7)$ & 0.764 & $0.5(0.08-6.5)$ & 0.592 \\
\hline Did not report & $1.3(0.4-3.6)$ & 0.677 & $1.2(0.4-3.6)$ & 0.694 & $2.1(0.6-6.8)$ & 0.236 & $1.6(0.5-5.8)$ & 0.447 & $1.3(0.3-5.0)$ & 0.745 & $1.5(0.3-6.9)$ & 0.585 \\
\hline \multicolumn{13}{|l|}{ Daily frequency } \\
\hline Once & 1 & - & 1 & - & 1 & - & 1 & - & 1 & - & 1 & - \\
\hline Twice & $1.4(0.4-5.2)$ & 0.604 & $1.6(0.4-6.4)$ & 0.479 & $1.7(0.8-3.5)$ & 0.153 & $1.7(0.8-3.7)$ & 0.162 & $0.3(0.07-1.1)$ & 0.060 & $0.3(0.08-1.5)$ & 0.149 \\
\hline Did not report & $0.8(0.05-11.3)$ & 0.835 & $0.8(0.05-13.3)$ & 0.883 & $4.6(0.8-27.9)$ & 0.095 & $3.7(0.6-26.1)$ & 0.177 & Empty & - & Empty & - \\
\hline \multicolumn{13}{|l|}{ Storage } \\
\hline Cupboard & 1 & - & 1 & & 1 & - & 1 & - & 1 & - & 1 & - \\
\hline Handbag & $1.0(0.5-2.2)$ & 1.000 & $1.0(0.4-2.2)$ & 0.964 & $1.1(0.5-2.5)$ & 0.782 & $1.4(0.6-3.1)$ & 0.478 & $0.2(0.05-1.0)$ & 0.054 & $0.2(0.05-1.0)$ & 0.055 \\
\hline Pill box & $0.4(0.09-1.5)$ & 0.166 & $0.3(0.08-1.4)$ & 0.131 & $0.9(0.2-3.7)$ & 0.816 & $1.2(0.3-5.5)$ & 0.809 & Empty & - & Empty & - \\
\hline Other & Empty & - & Empty & - & $3.5(0.3-40.3)$ & 0.312 & $1.7(0.1-22.8)$ & 0.678 & Empty & - & Empty & - \\
\hline Did not report & $1.0(0.2-5.2)$ & 1.000 & $1.1(0.2-6.3)$ & 0.931 & $2.3(0.5-11.1)$ & 0.284 & $2.3(0.4-12.2)$ & 0.336 & $1.9(0.3-11.3)$ & 0.470 & $2.3(0.3-16.9)$ & 0.430 \\
\hline
\end{tabular}

Note: All logistic regression analyses were performed at $95 \% \mathrm{Cl}$.

UOR, unadjusted odds ratios; AOR, adjusted odds ratios; $p$, probability value; Ref, reference; $\mathrm{Cl}$, confidence interval.

$*, p<0.05$.

more reports of treatment interruption whilst participants were on first-line treatment $(n=52)$. With respect to the reported treatment interruption whilst on second-line regimen, there was no distinct difference between the failing $(n=8)$ and suppressed $(n=5)$ groups. Both groups equally relied on themselves to remember to take their treatment ('naturally [I] remember taking my pills [VLF, woman, 31 years]; [I am] experienced on remembering my time' [VLS, man, 41 years]). Some of the reasons for interrupting treatment included stopping to take tuberculosis medication ('yes, interruption due to TB recurrence' [VLF, woman, 22 years]) and no medication availability whilst relocating
('I once interrupted my treatment due to the shortage of drugs as I relocated in South Africa and I did not have a proper transfer letter' [VLF, man, 38 years]). In the group with no reported second-line treatment interruption, more virologically suppressed participants $(n=21)$ listed using an alarm as a reminder. Several participants used the timeslots of popular local television programmes or the news $(n=14)$ as reminders to take their medication.

Table 4 presents the bivariate and multivariate logistic regression analyses for ART-taking behaviour. There was no association between participants' treatment-taking behaviour 
(i.e. medication alert, daily frequencies and pill storage) and VLF. However, participants who relied on their memory as a reminder to take medication (whilst on first-line regimen) were almost three times more likely to interrupt their firstline treatment than those who relied on an alarm (AOR: 2.6, $95 \% \mathrm{CI}=1.0-6.4, p=0.042$ ). There was no association found between medication alert or frequency of taking medication and second-line treatment interruption. Participants who used their handbag to store their medication were the least likely to experience second-line treatment interruption (OR: $0.2,95 \% \mathrm{CI}=0.05-1.0, p=0.054$ ).

\section{Recommendations from participants}

The study participants made 175 recommendations for improving adherence (see Table 5). Coformulation in single tablets, only needing to take one dose of medication daily (preferably at night) and education about being adherent were listed as the most effective mechanisms to improve adherence on second-line treatment. Some examples of recommendations from participants include the following:

'Education should be emphasised through adherence classes. Reinforce on the benefits of ART'. (VLS, female, 38 years old)

'Availing a single-dose treatment for the second-line patients would enable them to adhere to treatment. Further ongoing education would also help'. (VLF, female, 39 years old)

Other recommendations included the development of injectable ART ( $n=9$, seven women and two men) and the provision of psychosocial support (particularly related to poverty and ensuring food supply):

'Should consider addressing the psychosocial needs of patients on second line as they have to adhere to treatment but sometimes they do not have enough food to eat'. (VLF, female, 45 years old)

Single recommendations to improve adherence included treatment reminders $(n=1)$, additional counselling $(n=1)$

TABLE 5: Participants' perspectives on how adherence can be improved amongst second-line patients.

\begin{tabular}{lc} 
Recommendation & $\begin{array}{c}\text { Number of people citing the } \\
\text { recommendation }\end{array}$ \\
\hline Coformulation in single tablets & 55 \\
One dose & 46 \\
Education & 24 \\
No recommendations & 12 \\
Injection & 9 \\
Psychosocial support & 9 \\
Smaller pills & 7 \\
No side effects & 4 \\
Clinic operating times & 2 \\
Counselling & 1 \\
Decreased frequency of treatment ingestion & 1 \\
Follow-up & 1 \\
Liquid & 1 \\
More research needed & 1 \\
SMS reminders & 1 \\
Trust patients & 1 \\
\hline TOTAL & 175 \\
\hline
\end{tabular}

SMS, short message service. and minimising the number of times second-line regimen is taken per day $(n=1)$. One patient felt that healthcare workers trusting that their patients took their medication as prescribed would promote treatment adherence. Lastly, 12 participants did not have any recommendations (mainly because of not experiencing any pill-taking challenges), as shown in the following statement:

'The current regimen is fine with me, therefore, I will suggest no change'. (VLS, male, 41 years old)

\section{Discussion}

This study sought to describe and understand treatment adherence and possible treatment support interventions from patients receiving second-line ART.

It has been reported that relationship dynamics influence ART adherence and VLS in that being married or having a committed and supportive partner tended to foster an environment for better clinical outcomes in HIV-positive people. ${ }^{17,18}$ Studies from South Africa and the United Kingdom found that HIV-positive married individuals had better clinical outcomes compared to any other relationship status. ${ }^{19,20}$ Similarly, our study found that single and unmarried people living with their partners were more likely to be virally unsuppressed.

Not statistically significant but important for consideration in adherence strengthening, our study showed that being younger was a predictor of VLF, which was congruent with previous studies..$^{21,22,23}$ We noted VLS in those participants who resided further away from the health facilities. This is not in agreement with findings of studies conducted in Uganda, Ghana and Burkina Faso, ${ }^{24,25,26}$ which reported that individuals who resided closer to a health facility were more likely to seek healthcare.

Late disclosure may hinder adherence or treatment support and subsequently yield poor clinical outcomes. ${ }^{27}$ Whilst the majority of participants $(63 \%)$ disclosed their HIV status 1 week after diagnoses, about $28 \%$ took longer than 4 weeks to disclose. Early disclosure, particularly to a family member or partner, has been strongly associated with improved adherence. ${ }^{8,28,29}$ Disclosure to a family member or partner has been linked with adequate psychosocial support which in turn facilitates adherence to treatment. ${ }^{8,29,30,31,32}$ However, the findings of our study suggest that disclosure and dependence on a treatment supporter are likely not to produce desired adherence levels (and did not feature in the list of participant recommendations), indicating that disclosure and treatment support should be assessed in combination with other adherence strategies instead of as a single consideration or mechanism. ${ }^{33}$

Unsurprisingly, the more toxic the second-line multi-pill, and regimens requiring medication to be taken multiple times a day, were seen as significantly harder to take than a single tablet daily well-tolerated first-line regimen. These views were consistent with reports from other studies that attributed similar challenges to taking second-line regimen. . $^{34,35,36}$ 
Participants who did not interrupt ART mainly reported using an alarm as a reminder for taking their medication. This finding suggests the need to explore external reminder mechanisms for improving adherence in this setting, considering that about $15 \%$ of VLF participants reported not using any external reminders. Various studies have also found a trend towards better adherence amongst patients who used external reminders. ${ }^{37,38,39}$ In addition, our study showed that participants who used their handbags to store their medication were more likely to adhere to treatment. This finding is in line with other studies that have reported having a handbag to have pills all the time as the preferred ART storage by patients. ${ }^{40,41,42}$

Side effects are an important predictor of poor adherence, and cumulative toxicity associated with ART, especially in second-line regimens. ${ }^{43,44,45}$ We found that participants with VLF were more likely to have treatment-related side effects. Furthermore, those participants with side effects were more likely to be unemployed. Although this was not explored further in our study, various studies have reported that employed patients can manage their health and side effects better than their unemployed counterparts. ${ }^{46,47,48}$

Participants had ideas regarding drug formulation that may improve adherence. These included a fixed-dose combination, a dosage taken once a day and reducing the pill size. Furthermore, the participants suggested that education on the benefits of taking ART could improve adherence, whilst a few participants also suggested the implementation of injectable ART. Various studies have recommended similar strategies, ${ }^{49,50,51}$ with the effectiveness of some of these strategies being previously reported for first-line regimens. ${ }^{51,52}$

\section{Study limitations and strengths}

This study had several limitations. Firstly, the study relied on participants' self-reports, prompting a likelihood that socially desirable answers may have been provided. However, to control for this, information such as viral load, side effects and comorbidity was verified by checking participants' medical records as part of data quality checks for the study. Secondly, the clinical measure for adherence considered viral load only. Finally, the sample might be small for the results to be generalised to all patients receiving second-line ART. However, the direction and size of effect were generally consistent, suggesting that the study findings may be robust despite these limitations.

\section{Conclusion}

Participants on a second-line antiretroviral regimen had firm recommendations regarding improving adherence, largely focused on administration, reduced dosing and pill burden. The study results suggest the importance of improving patients' knowledge about treatment and adherence and motivation to continue ART use despite the persistence of side effects. Drug manufacturers and health programmers must consider such recommendations as they modify and implement new ART regimens and programmes. Lastly, treatment support interventions recommended in this study need to be tested in practice to determine their efficacy for large-scale implementation.

\section{Acknowledgements}

The authors would like to thank all the facilities and relevant health and research authorities from the City of Johannesburg for allowing the research team to engage in a partnership to strengthen health service delivery through technical assistance and research. They also thank the participants for sharing their experiences, views and recommendations, and Jean Claude Nkembi for his assistance with data collection and capture.

\section{Competing interests}

The authors declare that they have no competing interests.

\section{Authors' contributions}

S.B.G. and S.T.L.-E. designed the study and supervised the data extraction at the facilities. S.B.G. analysed the quantitative data and S.T.L.-E. analysed the qualitative data. S.B.G. interpreted the data and prepared the first draft of the manuscript, which was revised by S.T.L.-E. and W.D.F.V. All authors read and approved the final manuscript.

\section{Funding information}

This study was funded through the OPTIMIZE project. OPTIMIZE (AID-OAA-A-15-00069) is funded by the United States Agency for International Development (USAID) under the US President's Emergency Plan for AIDS Relief (PEPFAR). S.B.G. was supported by the Consortium for Advanced Research Training in Africa (CARTA). It is jointly led by the African Population and Health Research Center and the University of the Witwatersrand and funded by the Carnegie Corporation of New York (Grant No. B 8606.R02), Swedish International Development Cooperation Agency (SIDA) (Grant No. 54100113), the Developing Excellence in Leadership, Training and Science (DELTAS) Africa Initiative (Grant No. 107768/Z/15/Z) and Deutscher Akademischer Austauschdienst (DAAD). The DELTAS Africa Initiative is an independent funding scheme of the African Academy of Sciences' (AAS) Alliance for Accelerating Excellence in Science in Africa (AESA) and supported by the New Partnership for Africa's Development Planning and Coordinating Agency (NEPAD Agency) with funding from the Wellcome Trust (the United Kingdom [UK]) and the UK government.

\section{Data availability statement}

The data sets used or analysed in this study are available from the corresponding author upon reasonable request.

\section{Disclaimer}

The views and opinions expressed in this article are those of the authors and do not necessarily reflect the official policy or position of any affiliated agency of the authors. 


\section{References}

1. World Health Organization. Consolidated guidelines on the use of antiretrovira drugs for treating and preventing HIV infection: Recommendations for a public health approach [homepage on the Internet]. WHO Guidelines. 2013 public health approach homepage on the Internet]. WHO Guidelines. 2013
[cited 2019 Aug 14]. Available from: https://apps.who.int/iris/bitstream/ [cited 2019 Aug 14]. Available from: https://apps.who.int/
handle/10665/85321/9789241505727_eng.pdf?sequence $=1$

2. Fox MP, Berhanu R, Steegen $K$, et al. Intensive adherence counselling for HIVinfected individuals failing second-line antiretroviral therapy in Johannesburg,
South Africa. Trop Med Int Heal. 2016;21(9):1131-1137. https://doi.org/10.1111/ tmi.12741

3. Nieuwlaat R, Wilczynski N, Navarro $T$, et al. Interventions for enhancing medication adherence. Cochrane Database Syst Rev. 2014;11:1-538. https://doi. org/10.1002/14651858.CD000011.pub4

4. World Health Organization. Guideline on when to start antiretroviral therapy and on pre-exposure prophylaxis for HIV [homepage on the Internet]. World Health Organization; 2015 [cited 2019 Aug 14]. Available from: https://apps.who.int/iris/ bitstream/handle/10665/186275/9789241509565_eng.pdf?sequence $=1$

5. Goudge J, Ngoma B. Exploring antiretroviral treatment adherence in an urban setting in South Africa. J Public Health Policy. 2011;32:S52-S64. https://doi. org/10.1057/jphp.2011.22

6. Elliott JH, Lynen L, Calmy A, et al. Rational use of antiretroviral therapy in low-income and middle-income countries: Optimizing regimen sequencing and switching. AIDS. 2008;22(16):2053-2067. https://doi.org/10.1097/QAD.0b013e328309520d

7. Gross $R$, Zheng $L$, Rosa $A$ La, et al. Partner-based adherence intervention for second-line antiretroviral therapy (ACTG A5234): A multinational randomised
trial. Lancet HIV. 2(1):e12-19. https://doi.org/10.1016/S2352-3018(14)00007-1

8. Onoya $D$, Nattey $C$, Budgell $E$, et al. Predicting the need for third-line antiretroviral therapy by identifying patients at high risk for failing second-line antiretrovira therapy in South Africa. AIDS Patient Care STDS. 31(5):205-212. https://doi. org/10.1089/apc.2016.0291

9. Gill CJ, Hamer DH, Simon JL, Thea DM, Sabin LL. No room for complacency about adherence to antiretroviral therapy in sub-Saharan Africa. AIDS 2005;19(12):1243-1249. https://doi.org/10.1097/01.aids.0000180094.04652.3b

10. South African National Department of Health. National consolidated guidelines for the prevention of mother-to-child transmission of HIV (PMTCT) and the management of HIV in children, adolescents and adults [homepage on the Internet]. Health Policy. 2015 [cited 2019 Aug 20]. Available from: https://sahivsoc.org/Files/ ARTGuidelines15052015.pdf

11. Nnambalirwa M, Govathson C, Evans D, McNamara L, Maskew M, Nyasulu P. Markers of poor adherence among adults with HIV attending Themba Lethu HIV Clinic, Helen Joseph Hospital, Johannesburg, South Africa. Trans R Soc Trop Med Hyg. 2016;110(12):696-704. https://doi.org/10.1093/trstmh/trx003

12. World Health Organization. HIV drug resistance report 2017 [homepage on the Internet]. World Health Organization; 2017 [cited 2019 Aug 20]. Available from: https://apps.who.int/iris/bitstream/handle/10665/255896/9789241512831-eng. pdf?sequence $=1$

13. Hertogs $K$, Bloor S, De Vroey V, et al. A novel human immunodeficiency virus type 1 reverse transcriptase mutational pattern confers phenotypic lamivudine resistance in the absence of mutation 184V. Antimicrob Agents Chemother. 2000; 44(3):568-573. https://doi.org/10.1128/AAC.44.3.568-573.2000

14. Achappa B, Madi D, Bhaskaran U, Ramapuram JT, Rao S, Mahalingam S. Adherence to antiretroviral therapy among people living with HIV. N Am J Med Sci. 2013;5(3):220-223. https://doi.org/10.4103/1947-2714.109196

15. Pagès-Puigdemont $N$, Mangues MA, Masip $M$, et al. Patients' perspective of medication adherence in chronic conditions: A qualitative study. Adv Ther. 2016 medication adherence in chronic conditions: A qualitative st
33:1740-1754. https://doi.org/10.1007/s12325-016-0394-6

16. Laba TL, Lehnbom E, Brien J-A, Jan S. Understanding if, how and why nonadherent decisions are made in an Australian community sample: A key to sustaining medication adherence in chronic disease? Res Social Adm Pharm 2015;11(2):154-162. https://doi.org/10.1016/j.sapharm.2014.06.006

17. Conroy A, Leddy A, Johnson M, Ngubane T, Van Rooyen H, Darbes L. 'I told her this is your life': Relationship dynamics, partner support and adherence to antiretroviral therapy among South African couples. Cult Heal Sex. 2017;19(11):1239-1253. https://doi.org/10.1080/13691058.2017.1309460

18. Johnson MO, Dilworth SE, Taylor JM, Darbes LA, Comfort ML, Neilands TB. Primary relationships, HIV treatment adherence, and virologic control. AIDS Behav. 2012;16:1511-1521. https://doi.org/10.1007/s10461-011-0021-0

19. Robards J, Evandrou M, Falkingham J, Vlachantoni A. Marital status, health and mortality. Maturitas. 2012. https://doi.org/10.1016/j.maturitas.2012.08.007

20. Shisana O. South African national HIV prevalence, incidence and behaviour survey, 2012 [homepage on the Internet]. HSRC Press; 2014 [cited 2019 Aug 21] Available from: http://repository.hsrc.ac.za/bitstream/handle/20.500.11910/ 2490/8162.pdf? sequence=1\&isAllowed $=y$

21. Hadland SE, Milloy M-J, Kerr T, et al. Young age predicts poor antiretroviral adherence and viral load suppression among injection drug users. AIDS Patient Care STDS. 2012;26(5):274-280. https://doi.org/10.1089/apc.2011.0196

22. Bulage L, Ssewanyana I, Nankabirwa V, et al. Factors associated with virological nonsuppression among HIV-positive patients on antiretroviral therapy in Uganda, August
2014-July 2015. BMC Infect Dis. 2017;17(1):326. https://doi.org/10.1186/s128792017-July 2015

23. Mujugira A, Celum C, Tappero JW, Ronald A, Mugo N, Baeten JM. Younger age predicts failure to achieve viral suppression and virologic rebound among HIV-1infected persons in serodiscordant partnerships. AIDS Res Hum Retroviruses. 2016; 32(2):148-154. https://doi.org/10.1089/aid.2015.0296
24. Akullian AN, Mukose A, Levine GA, Babigumira JB. People living with HIV travel farther to access healthcare: A population-based geographic analysis from farther to access healthcare: A population-based geographic analysis from
rural Uganda. J Int AIDS Soc. 2016;19(1):20171. https://doi.org/10.7448/ IAS.19.1.20171

25. Buor D. Analysing the primacy of distance in the utilization of health services in the Ahafo-Ano South district, Ghana. Int J Health Plann Manage. 2003;18(4):293-311. https://doi.org/10.1002/hpm.729

26. Schoeps A, Gabrysch S, Niamba L, Sié A, Becher H. The effect of distance to healthcare facilities on childhood mortality in rural Burkina Faso. Am J Epidemiol. 2011;173(5):492-498. https://doi.org/10.1093/aje/kwq386

27. Cluver LD, Hodes RJ, Toska E, et al. 'HIV is like a tsotsi. ARVs are your guns': Associations between HIV-disclosure and adherence to antiretroviral treatment among adolescents in South Africa. AIDS. 2015;29 Suppl 1:S57-65. https://doi. org/10.1097/QAD.0000000000000695

28. Rochat TJ, Mkwanazi N, Bland R. Maternal HIV disclosure to HIV-uninfected children in rural South Africa: A pilot study of a family-based intervention. BMC Public Health. 2015;29 Suppl 1:S57-65. https://doi.org/10.1186/1471-245813-147

29. Wouters E, Van Loon F, Van Rensburg D, Meulemans H. Community support and disclosure of HIV serostatus to family members by public-sector antiretroviral treatment patients in the Free State Province of South Africa. AIDS Patient Care STDS. 2009;23(5):357-364. https://doi.org/10.1089/apc.2008.0201

30. Kunutsor S, Walley J, Katabira E, et al. Improving clinic attendance and adherence to antiretroviral therapy through a treatment supporter intervention in Uganda: A randomized controlled trial. AIDS Behav. 2011;15(8):1795-1802. https://doi. org/10.1007/s10461-011-9927-9

31. Nachega JB, Chaisson RE, Goliath R, et al. Randomized controlled trial of trained patient-nominated treatment supporters providing partial directly observed antiretroviral therapy. AIDS. 24(9):1273-1280. https://doi.org/10.1097/ QAD.0b013e328339e20e

32. Duwell MM, Knowlton AR, Nachega JB, et al. Patient-nominated, communitybased HIV treatment supporters: Patient perspectives, feasibility, challenges, and factors for success in HIV-infected South African adults. AIDS Patient Care STDS. 2013;27(2):96-102. https://doi.org/10.1089/apc.2012.0348

33. Kanters S, Park JJH, Chan K, et al. Interventions to improve adherence to antiretroviral therapy: A systematic review and network meta-analysis. Lancet HIV. 2017; 4(1):e31-e40. https://doi.org/10.1016/S2352-3018(16)30206-5

34. Mills EJ, Nachega JB, Bangsberg DR, et al. Adherence to HAART: A systematic review of developed and developing nation patient-reported barriers and facilitators. PLoS Med. 2006;3(11):e438. https://doi.org/10.1371/journal. pmed. 0030438

35. Ajose O, Mookerjee S, Mills EJ, Boulle A, Ford N. Treatment outcomes of patients on second-line antiretroviral therapy in resource-limited settings: A systematic review and meta-analysis. AIDS. 2012. https://doi.org/10.1097/QAD.0b013e328351f5b2

36. Cheng $Y$, Nickman NA, Jamjian $C$, et al. Predicting poor adherence to antiretroviral therapy among treatment-naïve veterans infected with human immunodeficiency virus. Medicine. 2018;97(2):e9495. https://doi.org/10.1097/ MD.0000000000009495

37. Petersen ML, Wang Y, Van der Laan MJ, Guzman D, Riley E, Bangsberg DR. Pillbox organizers are associated with improved adherence to HIV antiretroviral therapy and viral suppression: A marginal structural model analysis. Clin Infect Dis. 2007;45(7):908-915. https://doi.org/10.1086/521250

38. Azia IN, Mukumbang FC, Van Wyk B. Barriers to adherence to antiretroviral treatment in a regional hospital in Vredenburg, Western Cape, South Africa. South Afr J HIV Med. 2016;17(1):476. https://doi.org/10.4102/sajhivmed.v17i1.476

39. Nozaki I, Dube C, Kakimoto K, Yamada N, Simpungwe JB. Social factors affecting ART adherence in rural settings in Zambia. AIDS Care. 2011;23(7):831-838. https://doi.org/10.1080/09540121.2010.542121

40. Nassir Azmach N. Adherence to antiretroviral therapy and associated factors among adult ARV users in Arba Minch Hospital, Southern Ethiopia. Cent African Public Heal. 2017;3(2):19-26. https://doi.org/10.11648/j.cajph.20170302.12

41. Stawarz K, Rodríguez MD, Cox AL, Blandford A. Understanding the use of contextual cues: Design implications for medication adherence technologies that support remembering. Digit Heal. 2016; 1;2:2055207616678707. https:// doi.org/10.1177/2055207616678707

42. Nkomo G, Mosalo A, Thupayagale-Tshweneagae GB. Adherence to treatment and retention to care of adult patients on antiretroviral therapy. Afr J Nurs Midwifery. 2018;20(1):1-13. https://doi.org/10.25159/2520-5293/1590

43. Wasti SP, Simkhada P, Randall J, Freeman JV, Van Teijlingen E. Factors influencing adherence to antiretroviral treatment in Nepal: A mixed-methods study. PLoS One. 2012;7(5):e35547. https://doi.org/10.1371/journal.pone.0035547

44. Fonsah JY, Njamnshi AK, Kouanfack C, et al. Adherence to antiretroviral therapy (ART) in Yaoundé-Cameroon: Association with opportunistic infections, depression, ART regimen and side effects. PLoS One. 2017;12(1):e0170893. https://doi.org/10.1371/journal.pone.0170893

45. Johnson MO, Dilworth SE, Taylor JM, Neilands TB. Improving coping skills for selfmanagement of treatment side effects can reduce antiretroviral medication nonadherence among people living with HIV. Ann Behav Med. 2011;41(1):83-91. https://doi.org/10.1007/s12160-010-9230-4

46. Moyo F, Chasela C, Brennan AT, et al. Treatment outcomes of HIV-positive patients on first-line antiretroviral therapy in private versus public HIV clinics in Johannesburg, South Africa. Clin Epidemiol. 2016;8:37-47. https://doi. org/10.2147/CLEP.S93014 
47. Goodman N. The impact of employment on the health status and health care costs of working-age people with disabilities. LEAD Cent Policy Br. 2015.

48. Kilian R, Lauber C, Kalkan R, et al. The relationships between employment, clinical status, and psychiatric hospitalisation in patients with schizophrenia receiving either IPS or a conventional vocational rehabilitation programme. Soc Psychiatry Psychiatr Epidemiol. 2012:47(9):1381-1389. https://doi.org/10.1007/s00127011-0451-z

49. Barnett W, Patten $G$, Kerschberger B, et al. Perceived adherence barriers among patients failing second-line antiretroviral therapy in Khayelitsha, South Africa. South Afr J HIV Med. 2013;14(4):170-176. https://doi.org/10.4102/sajhivmed. 14i4.51
50. Scanlon ML, Vreeman RC. Current strategies for improving access and adherence to antiretroviral therapies in resource-limited settings. HIV/AIDS. 2013:5:1-17. https://doi.org/10.2147/HIV.S28912

51. Chaiyachati KH, Ogbuoji O, Price $M$, Suthar AB, Negussie EK, Bärnighausen T. Interventions to improve adherence to antiretroviral therapy: A rapid systematic review. AIDS. 2014; 28:S187-S204. https://doi.org/10.1097/ QAD.0000000000000252

52. Ridgeway K, Dulli LS, Murray KR, et al. Interventions to improve antiretroviral therapy adherence among adolescents in low- and middle-income countries: A systematic review of the literature. PLoS One. 2018;13(1):e0189770. https://doi. org/10.1371/journal.pone.0189770 